\title{
Acceleration of electrons by interacting CMEs
}

\author{
M. Vandas ${ }^{1}$ and D. Odstrcil ${ }^{2, \star}$ \\ 1 Astronomical Institute, Academy of Sciences of the Czech Republic, Boční II 1401, 14131 Praha 4, Czech Republic \\ ${ }^{2}$ University of Colorado/CIRES and NOAA/Space Environment Center, Boulder, CO 80305, USA \\ e-mail: Dusan.Odstrcil@sec.noaa.gov
}

Received 11 June 2003 / Accepted 18 December 2003

\begin{abstract}
There is growing observational evidence that interaction of coronal mass ejections (CMEs) near the Sun is a common phenomenon, particularly during the solar cycle's maximum years. Recently, we performed 2.5-D MHD simulations of the interaction between two magnetic flux ropes. The aim was to provide some qualitative picture of the shock-cloud and cloudcloud dynamic interactions that might be relevant to the interaction of CMEs. A sufficiently fast CME generates a shock wave that penetrates a slower CME. Enhanced magnetic field magnitudes in the flux rope and helical structure may favor acceleration of electrons at the shock by the fast-Fermi process since multiple encounters are possible and nearly perpendicular parts of the shock are more abundant. Using results of our MHD simulations, we numerically examine the efficiency of electron acceleration during the CME's interaction.
\end{abstract}

Key words. magnetohydrodynamics (MHD) - sun: coronal mass ejections - shock waves - sun: particle emission

\section{Introduction}

There is growing observational evidence that interactions of coronal mass ejections (CMEs) near the Sun are a common phenomenon. Originally, the interactions were identified as large enhancements in type II radio bursts by Gopalswamy et al. (2001). These authors conclude that the enhancement occurs when a bow shock of the faster CME penetrates the core of a slower one, thereby increasing the shock strength and accelerating electrons. Mutual interactions of CMEs became a subject of MHD simulation studies (Wu et al. 2002; Odstrcil et al. 2003).

Recently, we have performed 2.5-D MHD simulations of the interaction between two magnetic flux ropes representing CMEs or magnetic clouds (Odstrcil et al. 2003). The aim was to provide some qualitative picture of the shock-cloud and cloud-cloud dynamic interactions that might be relevant to the interaction of CMEs. A fast CME (flux rope) generates a shock wave which penetrates a slower CME. Enhanced magnetic field intensity and a helical structure of the field lines within the flux rope may favor acceleration of electrons by the shock drift mechanism at the shock (fast-Fermi process) (Wu 1984; Leroy \& Mangeney 1984). Nearly perpendicular parts of the shock cover larger areas within the leading, slower CME (than outside of the flux rope), and multiple encounters may be

Send offprint requests to: M. Vandas, e-mail: vandas@ig.cas.cz

* On leave from Ondřejov Observatory, Astronomical Institute, Academy of Sciences of the Czech Republic, Czech Republic. possible. The aim of this paper is to examine electron acceleration quantitatively in such a situation.

\section{Acceleration of electrons by the shock drift mechanism}

This mechanism is efficient at nearly perpendicular shocks (Wu 1984; Leroy \& Mangeney 1984; Vandas 1989; Krauss-Varban $\&$ Wu 1989). Such shock waves act as a fast moving magnetic mirror. Gyro-radii of supra-thermal electrons are small and the shock thickness cannot be neglected. Thus, a shock wave with non-zero thickness must be considered (Vandas 1994). Figure 1 shows a model of such a shock wave. Trajectories of two representative electrons are plotted; one was reflected, and the other was transmitted. Electrons drift due to grad- $B$ in a direction opposite to the induced electric field $\boldsymbol{E}=-(\boldsymbol{V} \times \boldsymbol{B})$ in the shockfront layer; thus, they gain energy (Fig. 1b). The amount of energy gained by the electron is approximately proportional to the time it has spent in the shock layer and, thus, is generally larger for reflected electrons. The angle $\theta_{\mathrm{Bn}}$ must be close to $90^{\circ}$ (within several degrees) in order for the acceleration to be significant.

\section{MHD simulations}

Using results of our numerical MHD simulations, we numerically examine the efficiency of electron acceleration during a propagation of a shock wave through a flux rope. For this 


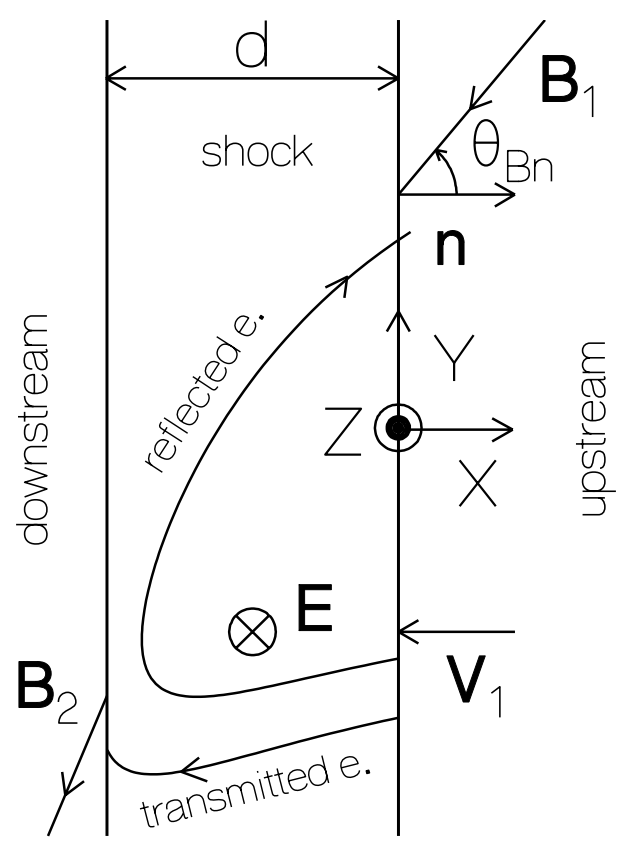

(a)

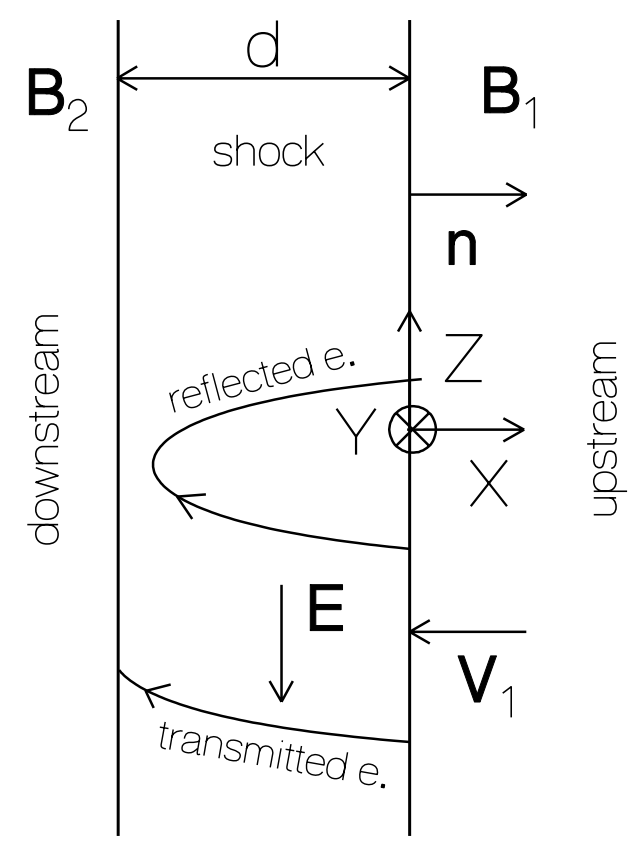

(b)

Fig. 1. Model of a plane shock wave in the shock wave rest frame in two coordinate projections: a) $X Y$, and b) $X Z$. The shock has the thickness $d$. The angle $\theta_{\mathrm{Bn}}$ is the angle between the upstream magnetic field $\boldsymbol{B}_{\mathbf{1}}$ and the shock normal $\boldsymbol{n}$. The upstream plasma velocity is $\boldsymbol{V}_{\mathbf{1}}$, the downstream magnetic field is $\boldsymbol{B}_{2}$. The induced electric field $\boldsymbol{E}=-\boldsymbol{V}_{\mathbf{1}} \times \boldsymbol{B}_{1}$ is supposed to be constant everywhere. Trajectories of reflected and transmitted electrons are shown. The scales of coordinates in the plot are not the same, the scale of $X$ being much smaller than the scales of $Y$ and $Z$ (which are identical).

purpose, we use a simpler configuration than the one used in the mentioned paper by Odstrcil et al. (2003). That is, we will now use a plane shock wave generated by a pressure pulse and that interacts with a single flux rope (Figs. 2 and 3). We assume that the interaction occurs at about 10 solar radii. The ambient values were put $3.4 \times 10^{4} \mathrm{~cm}^{-3}$ for the density, $10^{6} \mathrm{~K}$ for the temperature, and $0.7 \mu \mathrm{T}$ for the magnetic field strength. Further, the flux rope had the radius of 3 solar radii.

Two cases are considered. The flux rope in Case 1 (Fig. 2) has initially twice larger density than the background and its central magnetic field strength is four times larger than the background. The flux rope in Case 2 (Fig. 3) also has twice larger density but twice weaker magnetic field than the background value. As a result of different conditions, with different characteristic speed, the shock wave acquires very different shapes between cases, with implications for electron acceleration. The shock wave becomes weaker and faster inside the flux rope than outside in Case 1 and, facing outward, has a convex shape. The shock wave velocity is about $300 \mathrm{~km} \mathrm{~s}^{-1}$, and the jump is about 1.4 at the moment displayed in Fig. 2. The shock wave is stronger and slower inside the flux rope than outside in Case 2 and has a concave shape. The shock wave velocity is about $160 \mathrm{~km} \mathrm{~s}^{-1}$, and the jump is about 1.6 at the moment displayed in Fig. 3. In order to have comparable shock strengths for both cases, the initial pressure pulse in Case 1 was required to be stronger.

\section{Model shock wave}

The thickness of the shock wave in the MHD simulations was a few grid points, i.e., it was of the order of $10^{4} \mathrm{~km}$. The realistic thickness of the shock in the corona for our conditions is of the order of $1 \mathrm{~km}$ (Vandas \& Karlický 2000). Due to this huge difference, we had to create a model shock wave to make calculations of electron acceleration possible.

We tried to have a model shock wave that would preserve profiles of field quantities as close as possible to those from simulations. A planar shock wave in Fig. 1 has a constant $\theta_{\mathrm{Bn}}$. But our simulated shocks (Figs. 2 and 3 ) are curved. A curved shock wave is approximated by a similar model as shown in Fig. 1 but with $\theta_{\mathrm{Bn}}$ varying along the $Y$ axis (Krauss-Varban \& Burgess 1991; Vandas 1994). The origin of the XYZ system is identified with the nose of the shock wave (with coordinates $\left[x_{N}, y_{N}, z_{N}\right]$ where the upstream magnetic field is tangent to the shock front); then, the curved shock wave is projected to the planar layer tangent to the shock at the nose (Vandas 1995). In our cases with the $Y$ axis approximately parallel to the $y$ axis and the $Z$ axis parallel to the $z$ axis; the conditions, $Y \approx$ $y$ and $Z=z$, hold. The $X$ coordinate is measured along the shock normal at the given place. Shapes of the shock waves in Figs. 2 and 3 were approximated by a curve of the form, $x=$ $\alpha\left(y-y_{\mathrm{N}}\right)^{\beta}+x_{\mathrm{N}}$, and the shock normals were calculated using this form. Figure 4 shows how the angle $\theta_{\mathrm{Bn}}$ changes along the shock front for both cases. 


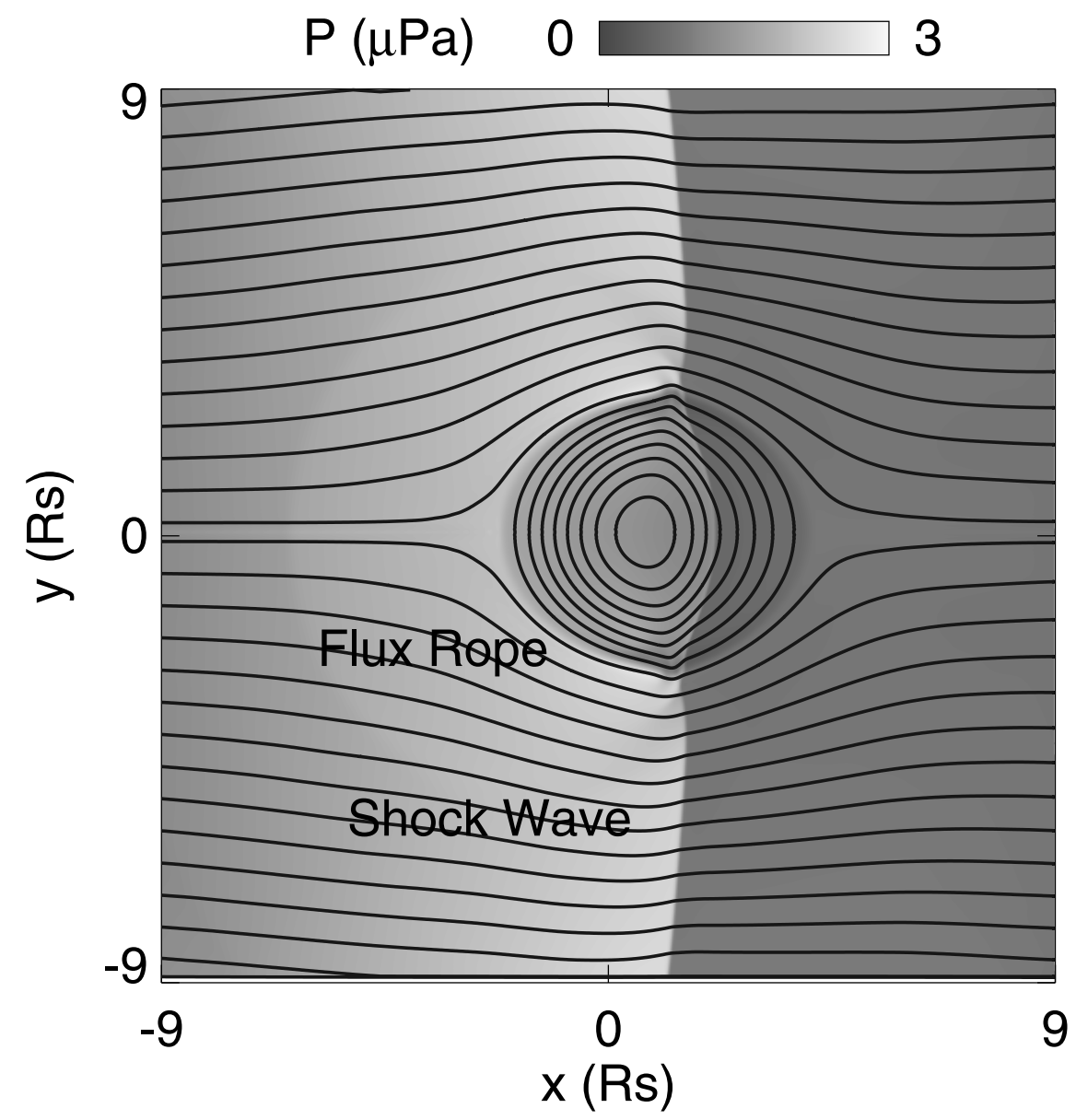

Fig. 2. A shock wave propagates through a flux rope in Case 1. Magnetic field lines are shown by the thick lines, the gray scale displays the plasma pressure $P$; the large plasma pressure gradients indicate the position of the shock. The figure shows a result of 2.5-D MHD numerical simulations: there was no dependency on the $z$ coordinate, but $z$ components of vectors were taken into account. Initial density within the flux rope is twice that in the ambient medium; also, the central flux rope's magnetic field magnitude is four times the ambient value. The coordinates $x$ and $y$ are in solar radii $(R \mathrm{~s})$.

Solid lines in Fig. 5 depict profiles of the magnetic and electric fields through the shock for Case 1 in a selected place (with $Y / \Delta y=10$ where $\theta_{\mathrm{Bn}}=83.8^{\circ}$ ) from MHD simulations. For our model plane shock wave we had to modify the profiles inside the shock layer slightly in order to meet requirements from the Maxwell equations, namely, that in the shock rest frame the components $B_{X}$ and $E_{Y}$ do not depend on $X ; B_{Y}$ and $E_{X}$ do not depend on $Y$; and $E_{Z}$ must be constant. Therefore, we made the following approximations for the model: (a) $B_{X}$ inside the shock was taken as its upstream value from the simulations for a given $Y$; (b) $E_{Y}$ inside the shock (in the shock rest frame) was set to an average value of $E_{Y}$ through the shock from the simulations for a given $Y$; (c) profiles of the model $B_{Y}$ and $E_{X}$ through the shock were the same for all $Y$ and coincide with the profiles from simulations at the nose; (d) $B_{Z}$ was calculated from the model $B_{X}$ and $B_{Y}$ and the magnitude $B$ from simulations and it retained the sign of $B_{Z}$ from simulations; (e) $E_{Z}$ was taken as an average value of $E_{Z}$ from simulations through the shock at the nose. The profiles of the model fields are shown by the dashed lines in Fig. 5; it is seen that they do not differ much from the simulation values (solid lines). Figure 6 shows that this model description holds also in a different situation; it compares the model fields with the values from simulations for Case 2 in the place with $Y / \Delta y=-5$ where $\theta_{\mathrm{Bn}}=89.4^{\circ}$.

\section{Calculation of electron acceleration}

Using the model field profiles and putting the thickness of the shock to be $1 \mathrm{~km}$, trajectories of electrons in this shock model were calculated in the guiding center approximation. Because of the specific topology of the upstream magnetic field lines, where "both upstream ends" of magnetic field lines intersect the shock, the overall acceleration scenario is different than, e.g., at planetary bow shocks (treated by, e.g., Vandas 1995). Electrons cannot escape and all are transmitted downstream. If an electron is reflected upstream, it will again interact with the shock because of the helical shape of the field line, because the line penetrates the shock front many times. So the upstream magnetic field lines create magnetic traps, and this fact increases the probability for a higher energy gain.

Figure 7 shows examples of four electron trajectories for four different initial conditions. All electrons had the same 


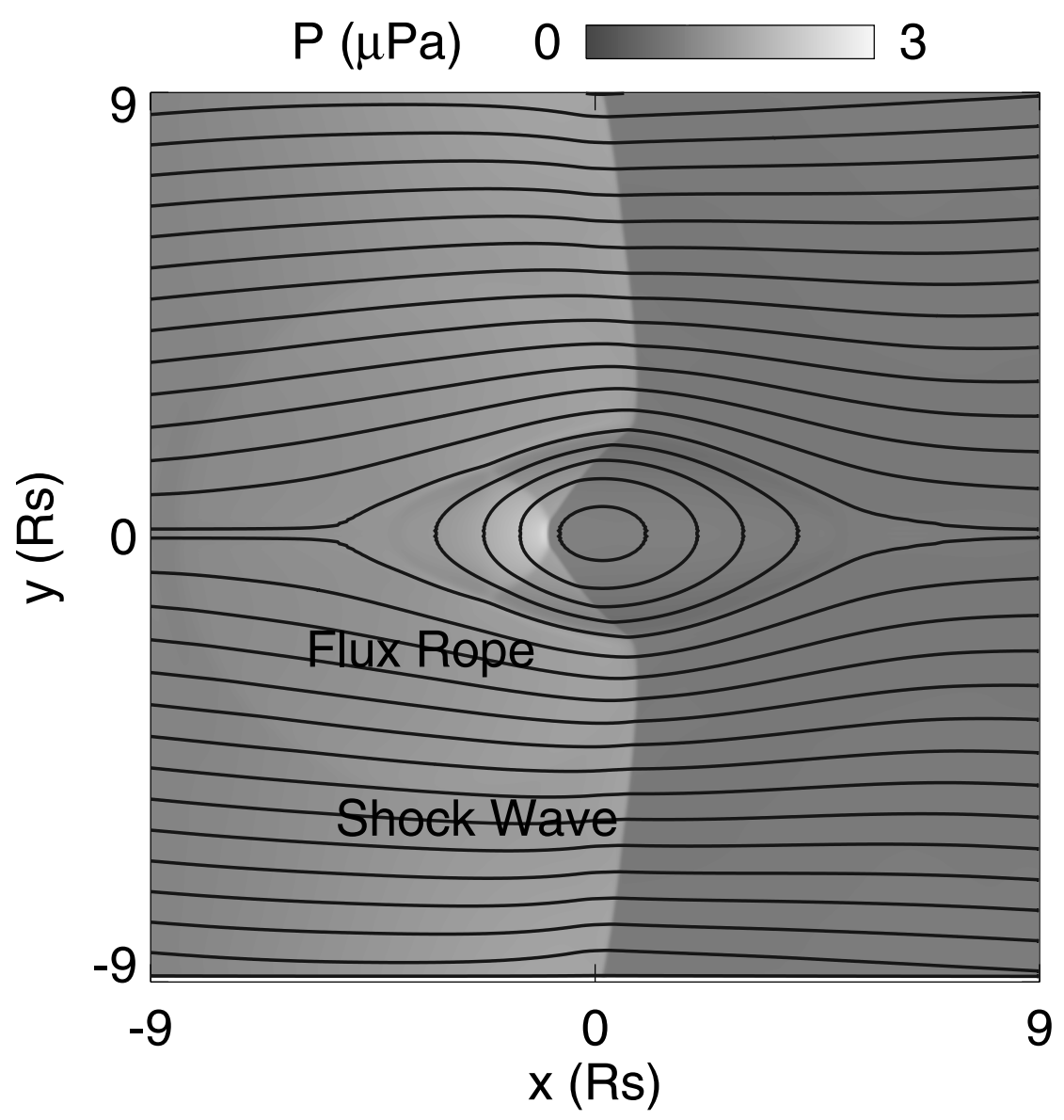

Fig. 3. A shock wave propagates through a flux rope in Case 2. The layout is similar to Fig. 2. As in Case 1, the flux rope's initial density is twice the ambient value, but the initial central magnetic field magnitude is one-half the background value.

initial energy $E_{\mathrm{i}}=1 \mathrm{keV}$. Figures $7 \mathrm{a}-\mathrm{c}$ are for Case 1 and electrons were injected at the same upstream position where $\theta_{\mathrm{Bn}} \approx 88.4^{\circ}$. Figure 7 a shows a trajectory of an electron with the initial pitch angle $\alpha_{\mathrm{i}}=87^{\circ}$ (pitch angle is the angle between the electron velocity and magnetic field vectors; an upstream electron velocity pointing towards the shock front has $\alpha_{\mathrm{i}}<90^{\circ}$ ). The electron was only transmitted downstream with the final values $E_{\mathrm{f}} \approx 1.5 \mathrm{keV}$ and $\alpha_{\mathrm{f}} \approx 71^{\circ}$. When the initial pitch angle is increased to $\alpha_{\mathrm{i}}=95^{\circ}$ (Fig. 7b), then the electron is reflected, moves upstream, returns to the shock and it is finally transmitted. Its final values are $E_{\mathrm{f}} \approx 2.3 \mathrm{keV}$ and $\alpha_{\mathrm{f}} \approx 49^{\circ}$. Figure $7 \mathrm{c}$ shows a somewhat extreme case when the initial electron pitch angle is $\alpha_{\mathrm{i}}=127^{\circ}$. The electron is reflected twice and then transmitted; its energy gain is thus very significant: the final values are $E_{\mathrm{f}} \approx 5.3 \mathrm{keV}$ and $\alpha_{\mathrm{f}} \approx 24^{\circ}$. Figure $7 \mathrm{~d}$ shows a trajectory of an electron for Case 2 . The electron with $\alpha_{\mathrm{i}}=74^{\circ}$ was injected in the upstream place where $\theta_{\mathrm{Bn}} \approx 87.5^{\circ}$. It was reflected and then transmitted. Its energy gain was only moderate because the reflection occurred with a relatively lower $\theta_{\mathrm{Bn}}$ : the final values were $E_{\mathrm{f}} \approx 1.5 \mathrm{keV}$ and $\alpha_{\mathrm{f}} \approx 69^{\circ}$.

Figure 8 compares the omnidirectional intensity of electrons at the downstream side of the shock just opposite to the nose for Cases 1 and 2 with the initial intensity. The initial distribution function of electrons is assumed to be a kappa distribution function with $\kappa=6$ (Vandas \& Karlický 2000). The intensity of accelerated supra-thermal electrons is enhanced by one order above the initial intensity. Despite of different shock shapes in our cases, the resulting intensity spectra of accelerated electrons are very similar.

\section{Conclusions}

We have performed a study of electron acceleration by the shock drift mechanism (fast Fermi process) at a shock penetrating a magnetic flux rope. Results of the 2.5-D numerical MHD simulations were combined with the theory of electron acceleration. The acceleration occurs only in small regions at the shock where the shock-front normal is nearly perpendicular to magnetic field lines. Bent field lines create local magnetic traps in these regions and these configurations are favorable to enhance electron energy gains. There are also global magnetic traps for a shock part within the flux rope. Electrons reflected by the shock may move far upstream along magnetic field lines, but helically-shaped line shapes inside the flux rope turned them eventually back again to the shock. However, the probability, that they meet again a nearly perpendicular region of the shock, is negligible. The same is true for shock-reflected 

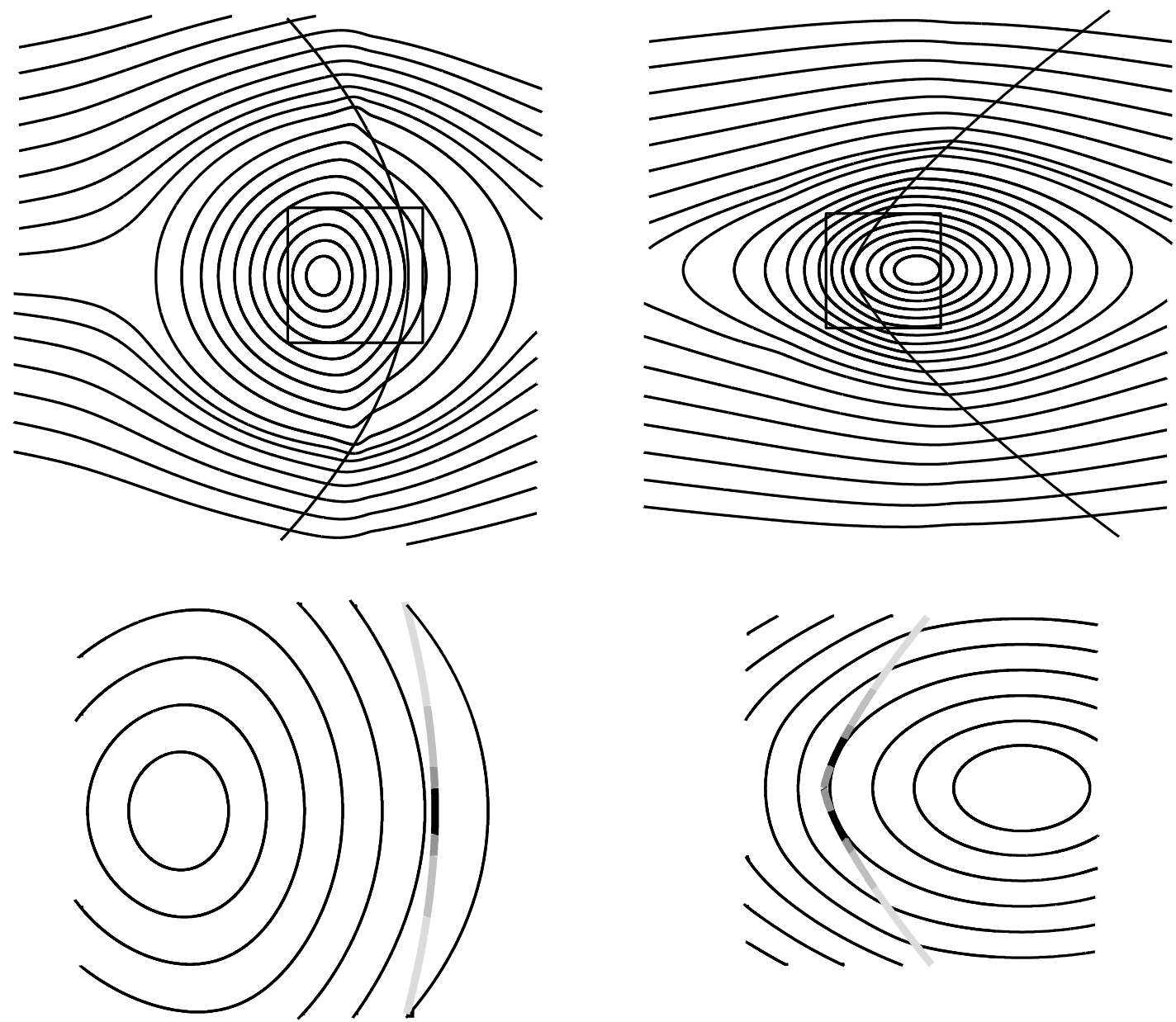

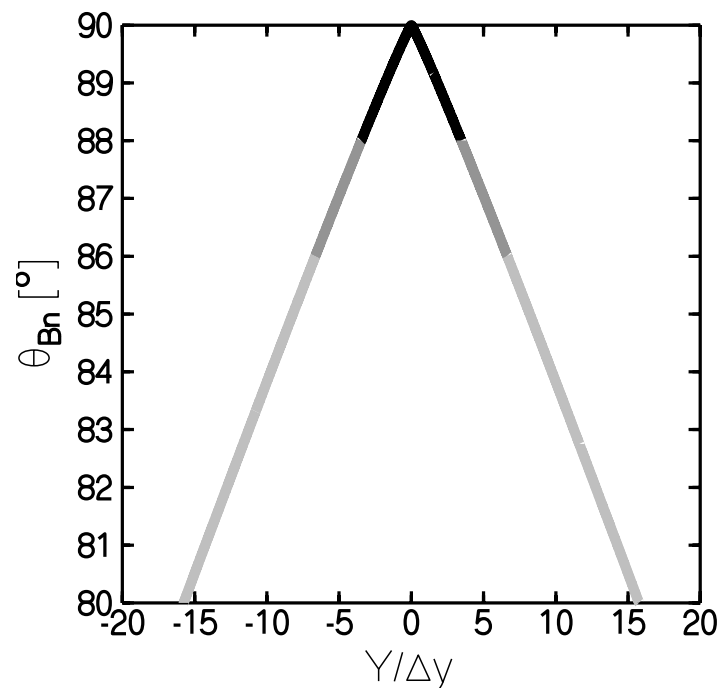

(a)

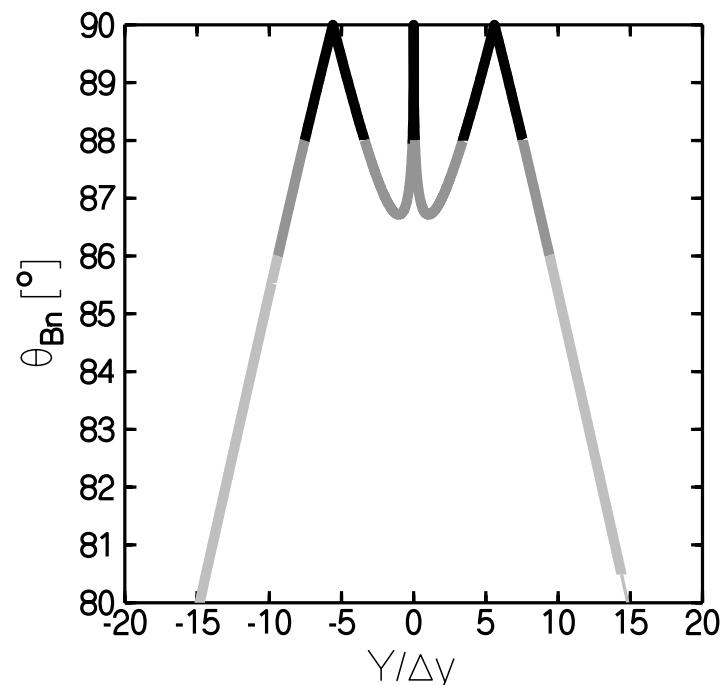

(b)

Fig. 4. Changes of the angle $\theta_{\mathrm{Bn}}$ along the shock surface for a) Case 1 and b) Case 2. Case 2 has more complex variations. Top plots show global views to the magnetic field lines (thin lines) and the shock shapes (thick lines) approximated by the expressions mentioned in the text for our two cases. Parts marked by boxes are zoomed in the middle plots, which display locations of a nearly perpendicular shock in the flux ropes. The shock drawn by the thick line is gray coded: the black line marks the interval of $\theta_{\mathrm{Bn}}$ between $88^{\circ}$ and $90^{\circ}$, and decreasing gray tones mark the intervals $86^{\circ}-88^{\circ}, 80^{\circ}-86^{\circ}$, and below $80^{\circ}$. The same coding is applied in the bottom graphs. $\Delta y$ is the grid size (e.g., Fig. 2 covers 480 grid points). 

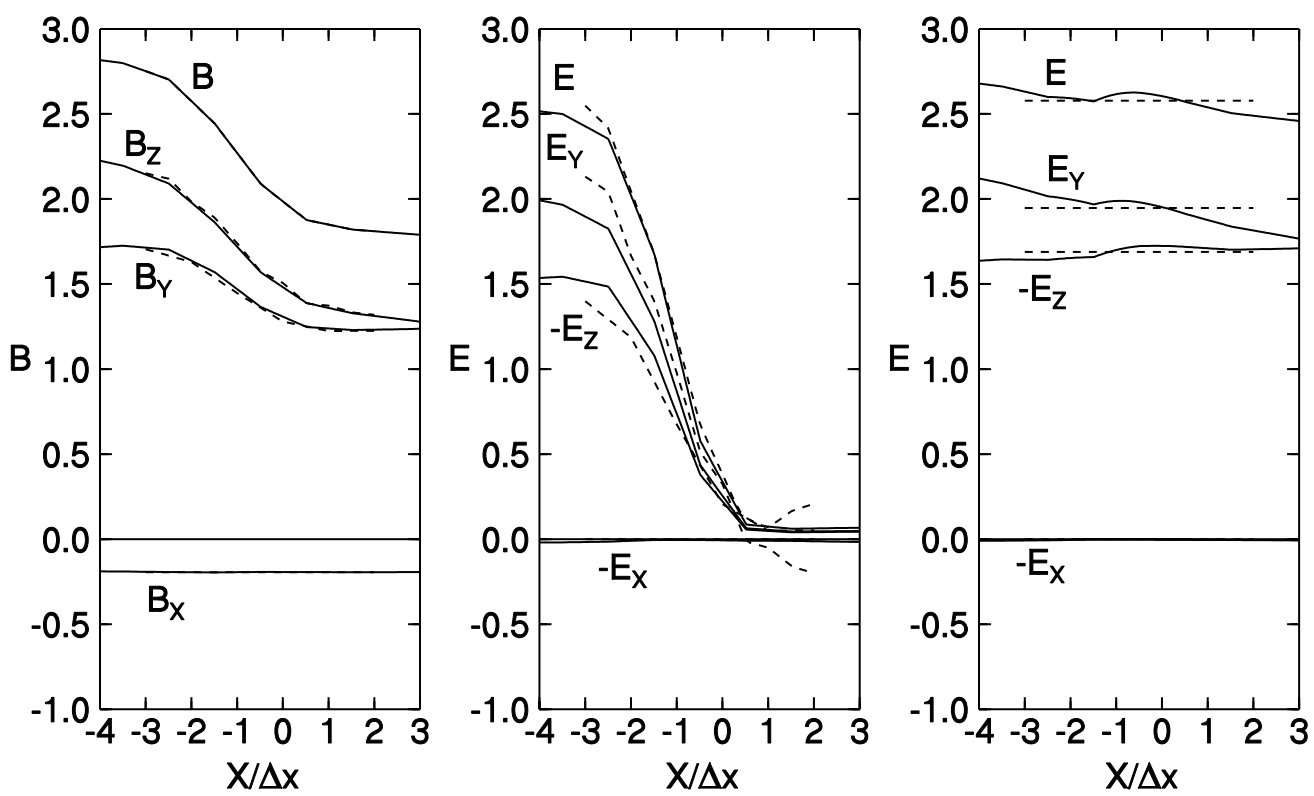

Fig. 5. The profiles of the magnetic (left panel) and electric (middle and right panels) fields through the shock (the value of 1.0 at the vertical axis corresponds to $1 \mu \mathrm{T}, 0.1 \mathrm{Vm}^{-1}$, and $0.2 \mathrm{Vm}^{-1}$ for the left, middle, and right panels, respectively). $\Delta x$ is the grid size $(\Delta x=\Delta y)$. The shock wave is located between $X / \Delta x=-2$ and 0 . The profiles in the left and middle panels are in the upstream plasma rest frame (frame of the MHD simulations), the profiles in the right panel are in the shock rest frame. Shown are components $B_{X}, B_{Y}, B_{Z},-E_{X}, E_{Y}$, and $-E_{Z} ; B$ and $E$ are field magnitudes. More explanation is in the text.
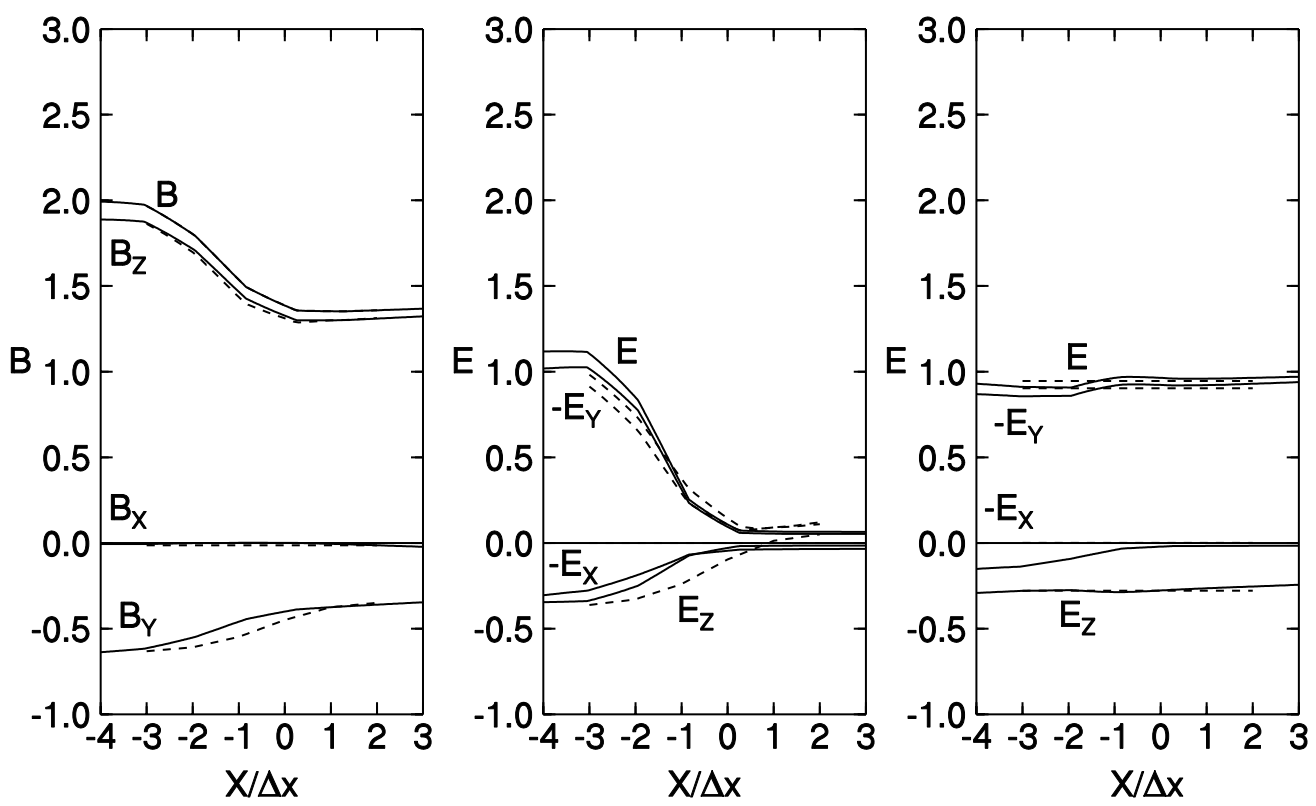

Fig. 6. The profiles of the magnetic and electric fields through the shock. The layout is the same as in Fig. 5.

electrons that move upstream along the flux rope and may be reflected back in its foot (at locations where the magnetic fluxrope loop may be tied to the photosphere) and return to the shock. The usage of a kappa function for the intial distribution function of electrons implicitly assumes that suprathermal particles are present prior to acceleration at perpendicular parts of the shock. These suprathermal particles could be produced by the first CME (its bow shock) or at parallel parts of the shock. If the latter statement is true, then the presence of both perpendicular and parallel geometries in a flux rope might be just as important as the existence of perpendicular shocks for accelerating particles.

Acknowledgements. This work was supported by project ME501 from MŠMT, by grant A3003003 and project S1003006 from the Academy of Sciences of the Czech Republic, and by DOD/AFOSR-MURI project. We thank to M. Dryer for discussions and careful reading of the manuscript. 


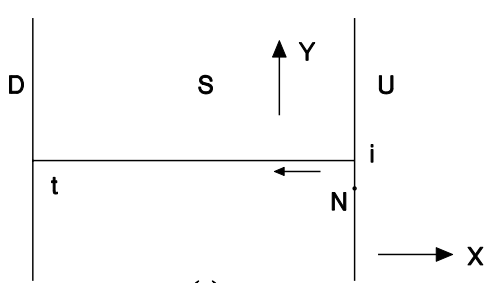

(a)

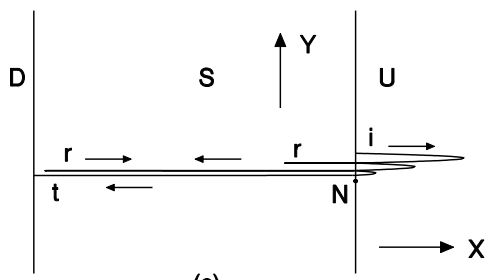

(c)

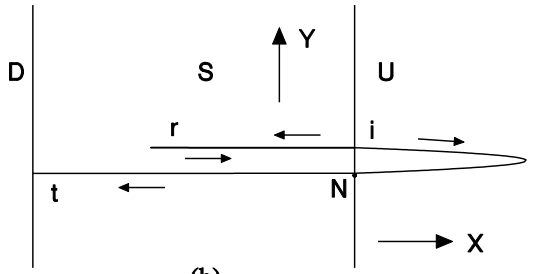

(b)

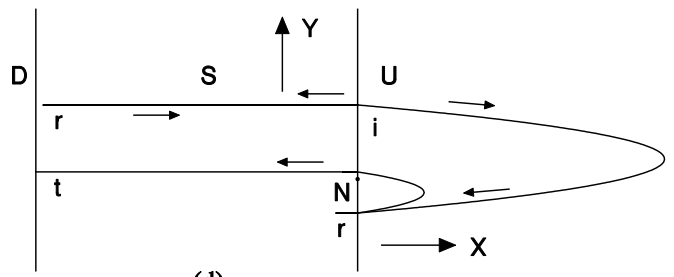

(d)

Fig. 7. Interactions of supra-thermal electrons with a shock inside a flux rope. The capital letters denote the regions around the shock, U and $D$ are the upstream and downstream regions, respectively, $\mathrm{S}$ is the shock layer; the bullets and $\mathrm{N}$ mark the place of the nose (the origin of the $X Y Z$ system). The curved lines are electron trajectories. The larger arrows show the directions of the axes, the smaller ones directions of electron motion. The letter i indicates the injection points of electrons, $\mathrm{r}$ and t label parts of trajectories where electrons are reflected or transmitted, respectively. For a purpose of a better readability, the $X$-scale inside the shock is much lower than the upstream $X$-scale and the latter is different for different plots; however, general proportions were kept, i.e., the largest upstream trajectory among the plots is also the largest in reality.

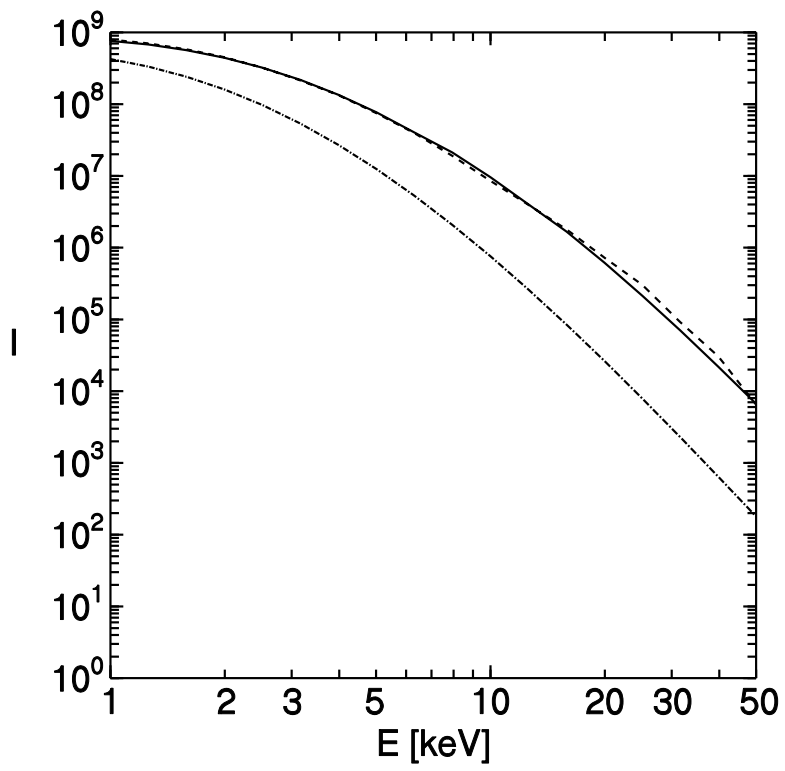

Fig. 8. Omnidirectional intensity of electrons (per unit energy) at the downstream side of the shock (in arbitrary units) as a function of energy for Case 1 (solid line) and Case 2 (dashed line) compared to the initial intensity (dash-dotted line).

\section{References}

Gopalswamy, N., Yashiro, S., Kaiser, M. L., Howard, R. A., \& Bougeret, J.-L. 2001, ApJ, 548, L91

Krauss-Varban, D., \& Wu, C. 1989, J. Geophys. Res., 94, 15367

Krauss-Varban, D., \& Burgess, D. 1991, J. Geophys. Res., 96, 143

Leroy, M., \& Mangeney, A. 1984, Ann. Geophys., 2, 449

Odstrcil, D., Vandas, M., Pizzo, V. J., \& MacNeice, P. J. 2003, in AIP Conf. Proc. 679, Solar Wind Ten (Melville, New York: AIP), 699

Vandas, M. 1989, Bull. Astron. Inst. Czech., 40, 189

Vandas, M. 1994, ApJS, 90, 583 40, 189

Vandas, M. 1995, J. Geophys. Res., 100, 23499

Vandas, M., \& Karlický, M. 2000, Sol. Phys., 197, 85

Wu, C. 1984, J. Geophys. Res., 89, 8857

Wu, S. T., Wang, A. H., \& Gopalswamy, N. 2002, in SOLMAG 2002, Proc. Magnetic Coupling of the Solar Atmosphere Euroconference \& IAU Coll. 188, ESA SP-505 (Noordwijk, The Netherlands: ESA), 227 